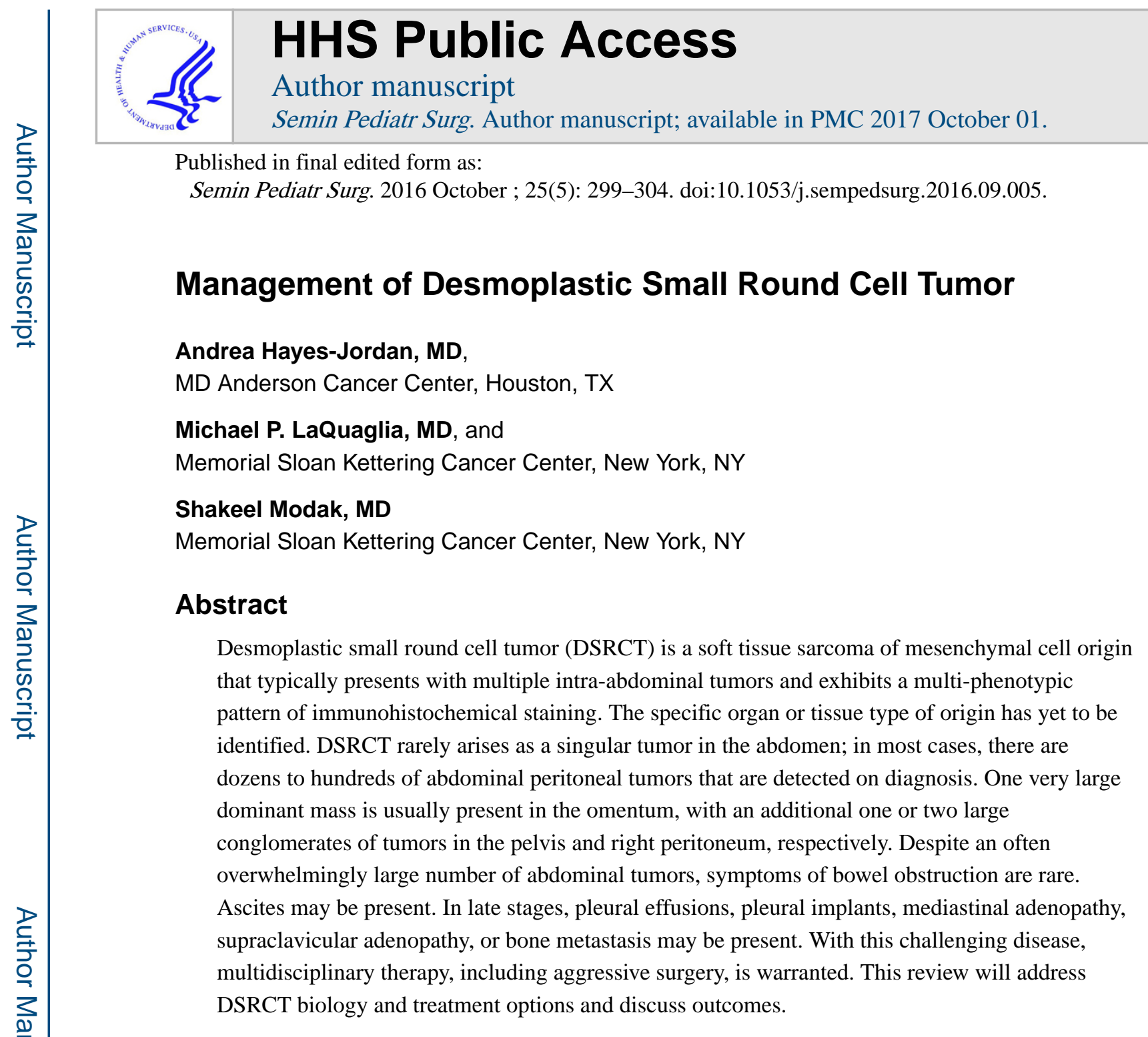

\title{
Introduction
}

Desmoplastic small round cell tumor (DSRCT) is a very recently described tumor, characterized in 1989 by Gerald and Rosai, who identified the EWS-WT1 translocation and fusion protein as pathognomonic. If this fusion protein cannot be identified in the tissue, the diagnosis of DSRCT cannot be made. DSRCT was a relatively unknown tumor that was considered by most clinicians to be an aggressive, rare, and lethal sarcoma. Categorizing the unique pathology and identifying any characteristic chromosomal translocations were of key importance to developing any treatment strategies ${ }^{1,2}$. Gerald and Rosai described the histologic appearance, which is characterized by nests of small round blue cells separated by desmoplastic stroma (Figure 1), and noted the immunohistochemical finding of multiphenotypic differentiation. Thus, DSCRT stains with desmin, cytokeratin, and S100, which

Corresponding author: Andrea Hayes-Jordan, MD, The University of Texas MD Anderson Cancer Center, Department of Surgical Oncology, 1400 Pressler St., Unit 1484, Houston, TX 77030, ahjordan@ mdanderson.org.

Publisher's Disclaimer: This is a PDF file of an unedited manuscript that has been accepted for publication. As a service to our customers we are providing this early version of the manuscript. The manuscript will undergo copyediting, typesetting, and review of the resulting proof before it is published in its final citable form. Please note that during the production process errors may be discovered which could affect the content, and all legal disclaimers that apply to the journal pertain. 
are mesenchymal, epithelial, and neural markers, respectively. Subsequent cytogenetic research by Ladanyi and Gerald identified a unique (11:22), (p13:q12) translocation and demonstrated that this translocation, which results in an active fusion protein involving the Ewing sarcoma (EWS) and Wilms tumor (WT-1) genes, is pathognomonic for DSCRT ${ }^{1-3}$. The confirmation of this translocation by percutaneous or open biopsy is now an essential part of the workup and is required to definitively establish the diagnosis of DSRCT. Prognosis for this disease is quite poor, with five-year overall survival estimated at only 15 to $30 \%{ }^{1-3}$. If the EWS translocation is not identified, then accurate diagnosis is quite challenging. Detection of an EWSR1-WT1 rearrangement and selective WT1 carboxyterminus immunoreactivity (characteristic of DSRCT) or dual immunoreactivity for the WT1 amino-terminus and carboxy-terminus (characteristic of WT) remain the most discriminating diagnostic tools ${ }^{4}$.

\section{Diagnosis and Staging}

DSRCT can present at an age ranging from 5 to 50 years, with a mean age at presentation of 22 years. Overall, about $85-90 \%$ of patients are male, but the proportion of females tends to be slightly higher among patients younger than 20 years at diagnosis. ${ }^{5}$ Large masses, in addition to visceral and parietal seeding of the peritoneum, are a typical presentation in DSRCT in more than 50\%. The reason a large tumor burden exists at diagnosis is that few symptoms are present until the peritoneal surfaces are infiltrated with tumor, overwhelming the peritoneum, and impairing resorption of peritoneal fluid, causing ascites. Abdominal distension and discomfort are the usual presenting symptoms. Patients can also have pain and constipation. Because of the sarcomatosis seen, these patients are considered stage 4 at diagnosis. It is rare for a patient to present with only one or two masses. However, this can occur when the DSCRT is found incidentally at the time of another operation, like hernia repair, or diagnostic radiologic exam for another entity.

Because of the frequently diffuse nature of the disease on presentation, a new staging system is being considered and is now being used on a trial basis by Hayes-Jordan and colleagues at MD Anderson Cancer Center in Houston, Texas. In this proposed staging system, stage 1 patients are those with limited disease, localized to one or two sites in the abdomen or one site elsewhere. Stage 2 patients are those with any amount of extensive peritoneal disease; Stage 3, with liver metastasis and peritoneal disease; and Stage 4, with peritoneal and liver disease, as well as disease outside the abdominal cavity, including in the lymph nodes. This staging system is under investigation and has not yet been validated.

\section{Imaging characteristics}

Computerized axial tomography (CT) is the most useful initial imaging study and should be performed with both gastrointestinal and intravenous contrast. Magnetic resonance imaging (MRI) is helpful in cases where pelvic and hepatic lesions are present. On CT or MRI, multiple peritoneal implants typically can be seen, which significantly increases the suspicion of DSRCT. The most common site of initial organ metastasis is usually the liver. The lungs, pleura and mediastinum are the next most common locations for metastasis. Lymph node enlargement in the groin and neck can also be seen. Therefore, positron 
emission tomography (PET) imaging may be a helpful adjunct to evaluate distant metastasis at the time of staging ${ }^{6}$.

On initial imaging, the observed extent of disease may include lesions throughout the peritoneal cavity, commonly in the omentum, right diaphragm, and pelvis (Figure 2). Disease spread to the splenic hilum and various small bowel and colon mesenteric implants is also common. Retroperitoneal disease, however, is very uncommon. In most cases, the disease seen on CT or MRI imaging actually underestimates disease extent, as metastases may develop as 1- to 2-millimeter 'sheets' of confluent tumor, which is a common intraoperative finding (Figure 3). Metastatic disease outside of the abdominal cavity may be found in the mediastinum, pleura, supradiphragmatic lymph nodes, lung, and bone.

\section{Chemotherapy}

Since its description in 1989 by Gerald and Rosai at Memorial Sloan Kettering Cancer Center, multimodality chemotherapy has been used for DSRCT. Ewing's type chemotherapy, aggressive surgery, tumor debulking, total abdominal radiation therapy, and high-dose chemotherapy followed by autologous stem cell rescue have all been used in the treatment of DSRCT, with little improvement in survival. Durable remissions remain rare ${ }^{7}$ Control of DSRCT with chemotherapy is most effective in children treated with Ewing's type chemotherapy, which has become the standard after efficacy with this regimen was demonstrated by Kushner et $\mathrm{al}^{7}$. This chemotherapy is based on the alkylating agents cyclophosphamide or ifosfamide, along with vincristine and doxorubicin alternating with ifosfamide and etoposide. This regimen was shown to have a favorable outcome in a multidisciplinary approach in 12 DSRCT patients ${ }^{7}$. This chemotherapy regimen was used in combination with aggressive surgical complete excision and post-operative whole abdominal radiation, providing improved survival. With a median follow-up of 22 months, the median disease-free survival was 19 months. The regimen can be quite toxic, and frequent admissions for fever and myelosuppression can be expected. An alternative, more tolerable outpatient regimen has been utilized that includes neoadjuvant vincristine, ifosfamide, dextrazoxane/doxorubicin, and etoposide ${ }^{8}$. This is followed by aggressive surgical excision and removal of all gross disease. Adjuvant therapy consists of radiotherapy (30Gy whole abdomen) and irinotecan and temozolomide for a total of 12 cycles. This regimen yielded a disease-free interval of approximately 2 years and a good quality of life with regular school attendance and participation in plan activities. This regimen was tolerable after surgery and radiotherapy ${ }^{8}$. Based on preclinical data indicating elevated expression of pro-angiogenic factors in DSRCT ${ }^{9}$, irinotecan and temozolomide in combination with bevacizumab-induced blockade of angiogenesis administered prior to Ewing's type chemotherapy are also being studied in a pilot study in newly diagnosed patients (clinicaltrials.gov NCT01189643). Attempts to improve outcomes with myeloablative chemotherapy with carboplatin, thiotepa and topotecan after debulking surgery have been unsuccessful ${ }^{10}$. Experience with other chemotherapeutic agents has mostly been anecdotal. 


\section{Surgical Therapy}

As mentioned, abdominal sarcomatosis is a common finding with tumor implants ranging

from $1 \mathrm{~mm}$ to $40 \mathrm{~cm}$ or more. Typically, omental disease is found in most patients in addition to peritoneal studding on the diaphragm, spleen, Morrison's pouch, abdominal wall peritoneum, small bowel mesentery, and almost certainly in the pelvis. Peritonectomies are required in these locations for effective complete gross resection and cytoreduction. Because DSRCT is generally responsive to systemic agents, the feasibility of complete surgical resection should not be assessed until the response to neoadjuvant chemotherapy has reached a plateau, which typically occurs after 4 to 6 months. It should be noted that the size of many lesions may not decrease with chemotherapy but, more commonly, there is a notable decrease in tumor vascularity. Malignant ascites usually responds well to chemotherapy and results in physiologic improvement. In a report on the impact of complete surgical resection of DSRCT, LaQuaglia and colleagues found a 3-year overall survival of 58\% with complete resection and $0 \%$ when resection was not done and the patients were treated with chemotherapy and radiotherapy alone ${ }^{5}$.

Complete surgical resection, including cytoreduction and hyperthermic intraperitoneal chemotherapy (HIPEC), is standard therapy for appendiceal carcinoma pseudomyxoma peritonei, and other diseases ${ }^{11-18}$, and multimodal therapy consisting of complete cytoreduction and HIPEC has been found to improve survival in many studies of carcinomatosis ${ }^{2}$ 19-25. Among patients with DSRCT, even after chemotherapeutic cytoreduction and surgical resection of gross, visible disease, microscopic residual disease is often present. Hence, HIPEC has been examined as a potentially effective adjunctive intraoperative strategy in this population. A phase 1 clinical trial of HIPEC in pediatric patients has demonstrated safety of HIPEC in children using cisplatin (Figure 4); the maximum tolerated dose was $100 \mathrm{mg} / \mathrm{m} 2$ with a dose-limiting toxicity of grade 3 renal failure $^{26}$. In a cohort of 26 DSRCT patients who underwent surgical resection and HIPEC after neoadjuvant chemotherapy, the completeness of cytoreduction determined outcome. Median survival of only 26 months was reached when incomplete resection was accompanied by HIPEC compared to 63 months, with complete resection ${ }^{27}$.

Recently, results from a phase 2 study of HIPEC in 20 pediatric sarcoma patients, including DSRCT, revealed superior survival results for patients with DSRCT compared to other sarcoma histologies ${ }^{28}$. There were no peri-operative mortalities and no reoperations. Transient leukopenia or thrombocytopenia was seen in $15 \%$ of patients. Thirty-five percent of patients experienced serious complications including, wound infections requiring drainage, urinary tract infections and enterocutaneous fistula (the latter in patients treated with abdominal radiation prior to HIPEC). (Operating time averages about 12 hours.) Presently, the question of whether the effects of HIPEC and complete resection are additive remains unresolved and the use of HIPEC should be protocol-based. Use of HIPEC in the case of disease outside the peritoneal cavity, presence of liver metastases, and extensive retroperitoneal or portal lymphadenopathy is probably not indicated.

The technique of cytoreduction, decision for cytoreduction and HIPEC in DSRCT is different from that done for adults with carcinomatosis. DSRCT is much more nodular and 
much less infiltrative than carcinoma, particularly in the area of the small bowel mesentery and pelvis. This allows for tangential resection of many lesions. Dissection of tumors from the jejunal and ileal mesentery peritoneum is most often feasible and can be complete without small bowel resection. Likewise, what appears to be pelvic tumor encasing ureters can be dissected free of the ureter, bladder and rectum in most circumstances (Figure 5). This is usually not the case with carcinomas ${ }^{29}$. Low anterior resection of the rectum, splenectomy, and segmental bowel resections may be required in selected cases.

In summary, DSRCT is a unique type of sarcoma for which improvements in treatment strategies are being made that have resulted in longer survival. Chemotherapy should be offered despite what may be extensive disease on imaging, since aggressive surgery to completely extirpate the disease may be possible if there is a response to chemotherapy.

\section{Radiotherapy}

Consolidative whole-abdomen radiotherapy at a dose of 30Gy, with or without a focal boost, was initially utilized after debulking surgery. However, significant hematological and gastrointestinal toxicities were observed ${ }^{30}$. The advent of intensity-modulated radiation therapy (IMRT) has resulted in a significant reduction in radiotherapy-related morbidity specifically gastrointestinal and hematologic toxicity ${ }^{31}$. However, groups at both MSKCC (IMRT after debulking surgery) and MD Anderson (IMRT after debulking surgery and HIPEC) have reported sub-optimal disease-free survival of $<1$ year ${ }^{31,32}$. Most relapses were intraperitoneal, suggesting that intraperitoneal therapy in addition to conventional surgery, chemotherapy and radiotherapy is necessary to improve outcome.

\section{Immunotherapy and Newer Therapeutic Approaches}

Ninety-six percent of DSRCT samples were found to exhibit notable cell-surface expression of the immunomodulatory molecule $\mathrm{B} 7 \mathrm{H} 3$, which has limited expression in normal tissue. The murine monoclonal antibody $8 \mathrm{H} 9$ targets $\mathrm{B} 7 \mathrm{H} 3$, and intraperitoneal administration of the radioimmunonjugate ${ }^{131} \mathrm{I}-8 \mathrm{H} 9$ has been investigated in a phase I trial in 34 patients with DSRCT at MSKCC (Clinicaltrials.gov \#NCT01099644). Preliminary data on the first 13 patients demonstrated that treatment was well tolerated, and 6 of 7 patients treated after surgical debulking remained in remission at a median of 11 months post-therapy. Absorbed radiation doses to vital organs were well below tolerable levels. Pre-treatment with ${ }^{124} \mathrm{I}-8 \mathrm{H} 9$ enabled investigation of biodistribution using serial PET scans and detailed pharmacokinetic data ${ }^{33}$. Based on these encouraging observations, a phase II study combining 8H9-mediated radioimmunotherapy with external beam radiotherapy will be initiated in 2016. Other DSRCT antigens that have been targeted clinically in small numbers of patients include GD2 (via murine antibody $3 \mathrm{~F} 8$ and the anti-idiotypic antibody A1G4) ${ }^{34}$ and IGF1R (via the humanized antibody ganitumab) ${ }^{35}$. Other pathways being considered for therapeutic targeting include $\mathrm{mTOR}^{36}$ and androgen receptors ${ }^{37}$. One of the most salient clinical features of DSRCT is male predominance. The role of androgens in DSRCT was studied and reported in $2007^{37}$. A significant androgen receptor (AR) immunostaining pattern was found in 10 of 27 DSRCT tumor studied and was confirmed by Western blot in two patients. The functional status of the AR was demonstrated by stimulation of basal in vitro growth by 
supplemental testosterone and inhibition of basal and testosterone stimulated growth by flutamide. These results suggested that DSRCT may up or downregulate AR possibly for survival purposes as a growth factor receptor. Of six heavily pretreated patients with positive AR status treated with a combination of androgen blockade (AR blocker and biculatamide preceding Lupron check this please), one each had partial response, minor response and stable disease, although responses appeared to be short-lived ${ }^{37}$.

\section{Summary}

DSRCT is a relatively recently described, aggressive, malignant tumor affecting mostly males in adolescence and young adulthood. Chemotherapy, resection, and radiation therapy form the basis of present treatment. HIPEC and intra-peritoneal antibody instillation are newer approaches. Patient outcomes are presently unacceptable and further progress will require the development of new research-based therapies.

\section{References}

1. Gerald WL, Ladanyi M, de Alava E, et al. Clinical, pathologic, and molecular spectrum of tumors associated with $\mathrm{t}(11 ; 22)(\mathrm{p} 13 ; \mathrm{q} 12)$ : desmoplastic small round-cell tumor and its variants. J Clin Oncol. 1998; 16:3028-3036. [PubMed: 9738572]

2. Park BJ, Alexander HR, Libutti SK, et al. Treatment of primary peritoneal mesothelioma by continuous hyperthermic peritoneal perfusion (CHPP). Ann Surg Oncol. 1999; 6:582-590. [PubMed: 10493628]

3. Ladanyi M, Gerald W. Fusion of the EWS and WT1 genes in the desmoplastic small round cell tumor. Cancer Res. 1994; 54:2837-2840. [PubMed: 8187063]

4. Arnold MA, Schoenfield L, Limketkai BN, Arnold CA. Diagnostic pitfalls of differentiating desmoplastic small round cell tumor (DSRCT) from Wilms tumor (WT): overlapping morphologic and immunohistochemical features. The American journal of surgical pathology. 2014; 38:12201226. [PubMed: 24832162]

5. Lal DR, Su WT, Wolden SL, Loh KC, Modak S, La Quaglia MP. Results of multimodal treatment for desmoplastic small round cell tumors. J Pediatr Surg. 2005; 40:251-255. [PubMed: 15868593]

6. Zhang WD, Li CX, Liu QY, Hu YY, Cao Y, Huang JH. CT, MRI, and FDG-PET/CT imaging findings of abdominopelvic desmoplastic small round cell tumors: Correlation with histopathologic findings. Eur J Radiol. 2010

7. Kushner BH, LaQuaglia MP, Wollner N, et al. Desmoplastic small round-cell tumor: prolonged progression-free survival with aggressive multimodality therapy. J Clin Oncol. 1996; 14:1526-1531. [PubMed: 8622067]

8. Aguilera D, Hayes-Jordan A, Anderson P, Woo S, Pearson M, Green H. Outpatient and home chemotherapy with novel local control strategies in desmoplastic small round cell tumor. Sarcoma. 2008; 2008:261589. [PubMed: 18566684]

9. Magnan HD, Chou T, La Quaglia MP, Gerald W, Ladanyi M, Merchant TE. Elevated expression of VEGFR-2 and VEGFA in desmoplastic small round cell tumor (DSRCT) and activity of bevacizumab and irinotecan in a xenograft model of DSRCT. J Clin Oncol. 2009; 27:10016.

10. Forlenza CJ, Kushner BH, Kernan N, et al. Myeloablative chemotherapy with autologous stem cell transplant for desmoplastic small round cell tumor. Sarcoma. 2015; 2015:269197. [PubMed: 25945075]

11. Glehen O, Kwiatkowski F, Sugarbaker PH, et al. Cytoreductive surgery combined with perioperative intraperitoneal chemotherapy for the management of peritoneal carcinomatosis from colorectal cancer: a multi-institutional study. J Clin Oncol. 2004; 22:3284-3292. [PubMed: 15310771]

12. Sugarbaker PH. A curative approach to peritoneal carcinomatosis from colorectal cancer. Semin Oncol. 2005; 32:S68-S73. [PubMed: 16399436] 
13. Sugarbaker PH, Stuart OA, Yoo D. Strategies for management of the peritoneal surface component of cancer: cytoreductive surgery plus perioperative intraperitoneal chemotherapy. J Oncol Pharm Pract. 2005; 11:111-119. [PubMed: 16390599]

14. Sugarbaker PH, Jablonski KA. Prognostic features of 51 colorectal and 130 appendiceal cancer patients with peritoneal carcinomatosis treated by cytoreductive surgery and intraperitoneal chemotherapy. Annals of surgery. 1995; 221:124-132. [PubMed: 7857141]

15. Glehen O, Gilly FN, Sugarbaker PH. New perspectives in the management of colorectal cancer: what about peritoneal carcinomatosis? Scand J Surg. 2003; 92:178-179. [PubMed: 12841562]

16. Gough DB, Donohue JH, Schutt AJ, et al. Pseudomyxoma peritonei. Long-term patient survival with an aggressive regional approach. Annals of surgery. 1994; 219:112-119. [PubMed: 8129481]

17. Sugarbaker PH, Welch LS, Mohamed F, Glehen O. A review of peritoneal mesothelioma at the Washington Cancer Institute. Surg Oncol Clin N Am. 2003; 12:605-621. xi. [PubMed: 14567020]

18. Glehen O, Mithieux F, Osinsky D, et al. Surgery combined with peritonectomy procedures and intraperitoneal chemohyperthermia in abdominal cancers with peritoneal carcinomatosis: a phase II study. J Clin Oncol. 2003; 21:799-806. [PubMed: 12610177]

19. Yan TD, Edwards G, Alderman R, Marquardt CE, Sugarbaker PH. Morbidity and mortality assessment of cytoreductive surgery and perioperative intraperitoneal chemotherapy for diffuse malignant peritoneal mesothelioma--a prospective study of 70 consecutive cases. Ann Surg Oncol. 2007; 14:515-525. [PubMed: 17031722]

20. Yan TD, Welch L, Black D, Sugarbaker PH. A systematic review on the efficacy of cytoreductive surgery combined with perioperative intraperitoneal chemotherapy for diffuse malignancy peritoneal mesothelioma. Ann Oncol. 2007; 18:827-834. [PubMed: 17130182]

21. de Bree E, Romanos J, Michalakis J, et al. Intraoperative hyperthermic intraperitoneal chemotherapy with docetaxel as second-line treatment for peritoneal carcinomatosis of gynaecological origin. Anticancer Res. 2003; 23:3019-3027. [PubMed: 12926156]

22. Sugarbaker PH, Alderman R, Edwards G, et al. Prospective morbidity and mortality assessment of cytoreductive surgery plus perioperative intraperitoneal chemotherapy to treat peritoneal dissemination of appendiceal mucinous malignancy. Ann Surg Oncol. 2006; 13:635-644. [PubMed: 16523363]

23. Farma JM, Pingpank JF, Libutti SK, et al. Limited survival in patients with carcinomatosis from foregut malignancies after cytoreduction and continuous hyperthermic peritoneal perfusion. $\mathrm{J}$ Gastrointest Surg. 2005; 9:1346-1353. [PubMed: 16332493]

24. Kunisaki C, Shimada H, Akiyama H, et al. Therapeutic outcomes of continuous hyperthermic peritoneal perfusion against advanced gastric cancer with peritoneal carcinomatosis. Hepatogastroenterology. 2006; 53:473-478. [PubMed: 16795995]

25. Feldman AL, Libutti SK, Pingpank JF, et al. Analysis of factors associated with outcome in patients with malignant peritoneal mesothelioma undergoing surgical debulking and intraperitoneal chemotherapy. J Clin Oncol. 2003; 21:4560-4567. [PubMed: 14673042]

26. Hayes-Jordan A, Green H, Ludwig J, Anderson P. Toxicity of hyperthermic intraperitoneal chemotherapy (HIPEC) in pediatric patients with sarcomatosis/carcinomatosis: early experience and phase 1 results. Pediatric blood \& cancer. 2012; 59:395-397. [PubMed: 22492588]

27. Hayes-Jordan A, Green HL, Lin H, et al. Complete cytoreduction and HIPEC improves survival in desmoplastic small round cell tumor. Ann Surg Oncol. 2014; 21:220-224. [PubMed: 24046124]

28. Hayes-Jordan, A., Green, H., Xiao, LC., Fournier, K., Huh, W., Herzog, C., Ludwig, J., McAleer, M., Anderson, P. American Pediatric Surgical Association Annual Meeting. Fort Lauderdale, FL: 2015. Desmoplastic Small Round Cell Tumor treated with cytoreductive surgery and Hyperthermic Intraperitoneal Chemotherapy: Results of a phase 2 trial [abstract].

29. Hayes-Jordan A, Green H, Lin H, et al. Cytoreductive surgery and Hyperthermic Intraperitoneal Chemotherapy (HIPEC) for children, adolescents, and young adults: the first 50 cases. Ann Surg Oncol. 2015; 22:1726-1732. [PubMed: 25564159]

30. Goodman KA, Wolden SL, La Quaglia MP, Kushner BH. Whole abdominopelvic radiotherapy for desmoplastic small round-cell tumor. International journal of radiation oncology, biology, physics. 2002; 54:170-176. 
31. Desai NB, Stein NF, LaQuaglia MP, et al. Reduced toxicity with intensity modulated radiation therapy (IMRT) for desmoplastic small round cell tumor (DSRCT): an update on the whole abdominopelvic radiation therapy (WAP-RT) experience. International journal of radiation oncology, biology, physics. 2013; 85:e67-e72.

32. Osborne EM, Briere TM, Hayes-Jordan A, et al. Survival and toxicity following sequential multimodality treatment including whole abdominopelvic radiotherapy for patients with desmoplastic small round cell tumor. Radiotherapy and oncology : journal of the European Society for Therapeutic Radiology and Oncology. 2016; 119:40-44. [PubMed: 26527430]

33. Modak S, La Quaglia MP, Carrasquillo JA, et al. Intraperitoneal radioimmunotherapy (RIT) for desmoplastic small round cell tumor (DSRCT): Initial results from a phase I trial. J Clin Oncol. 2013; 31:3033.

34. Modak S, Gerald W, Cheung NK. Disialoganglioside GD2 and a novel tumor antigen: potential targets for immunotherapy of desmoplastic small round cell tumor. Medical and pediatric oncology. 2002; 39:547-551. [PubMed: 12376975]

35. Tap WD, Demetri G, Barnette P, et al. Phase II study of ganitumab, a fully human anti-type-1 insulin-like growth factor receptor antibody, in patients with metastatic Ewing family tumors or desmoplastic small round cell tumors. J Clin Oncol. 2012; 30:1849-1856. [PubMed: 22508822]

36. Thijs AM, van der Graaf WT, van Herpen CM. Temsirolimus for metastatic desmoplastic small round cell tumor. Pediatric blood \& cancer. 2010; 55:1431-1432. [PubMed: 20730888]

37. Fine RL, Shah SS, Moulton TA, et al. Androgen and c-Kit receptors in desmoplastic small round cell tumors resistant to chemotherapy: novel targets for therapy. Cancer chemotherapy and pharmacology. 2007; 59:429-437. [PubMed: 16896931] 


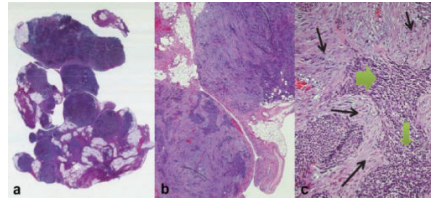

Figure 1.

Histologic sections of DSRCT from an omental biopsy, shown at low (panel a: $5 \times$, panel b: $20 \times$ ) and (c) high (40x) magnification. In panel c, nests of small round blue cells (filled arrow) interdigitate between bands of fibrous stroma (line arrow). 

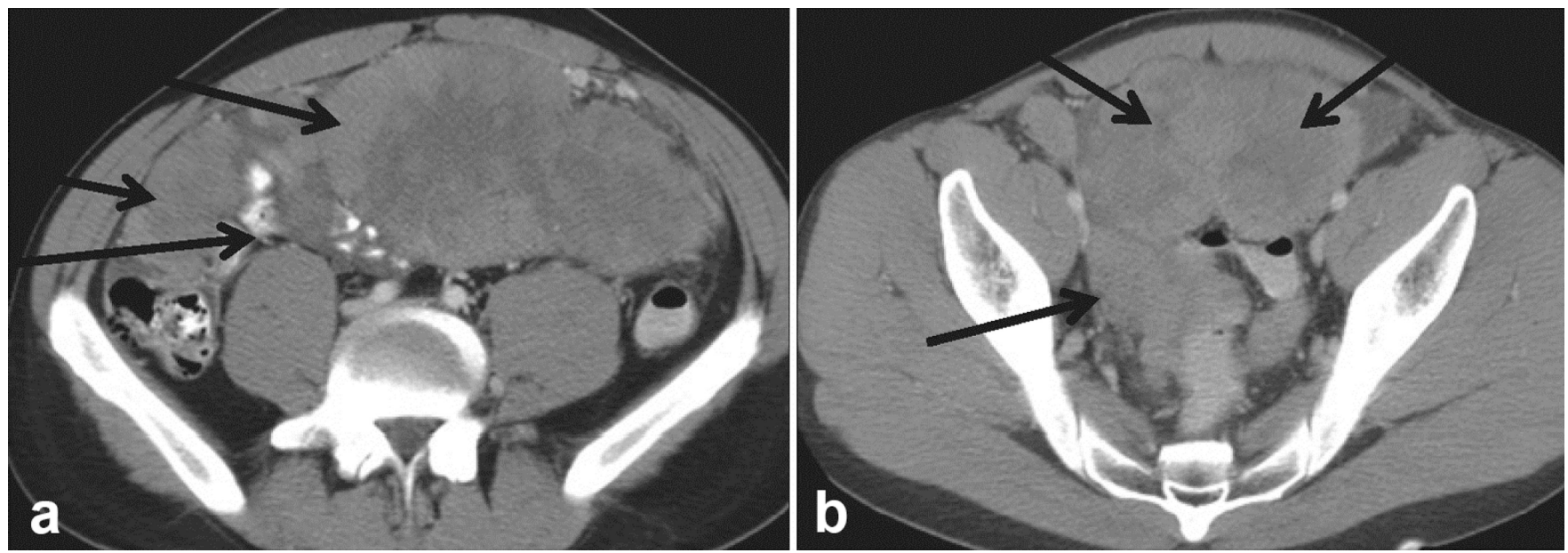

Figure 2.

Panel (a) shows a large omental mass in a newly diagnosed patient with DSRCT. Panel (b) shows a pelvic, paravesical mass, large and lobulated. Pelvic tumors are very typical of DSRCT sarcomatosis. 

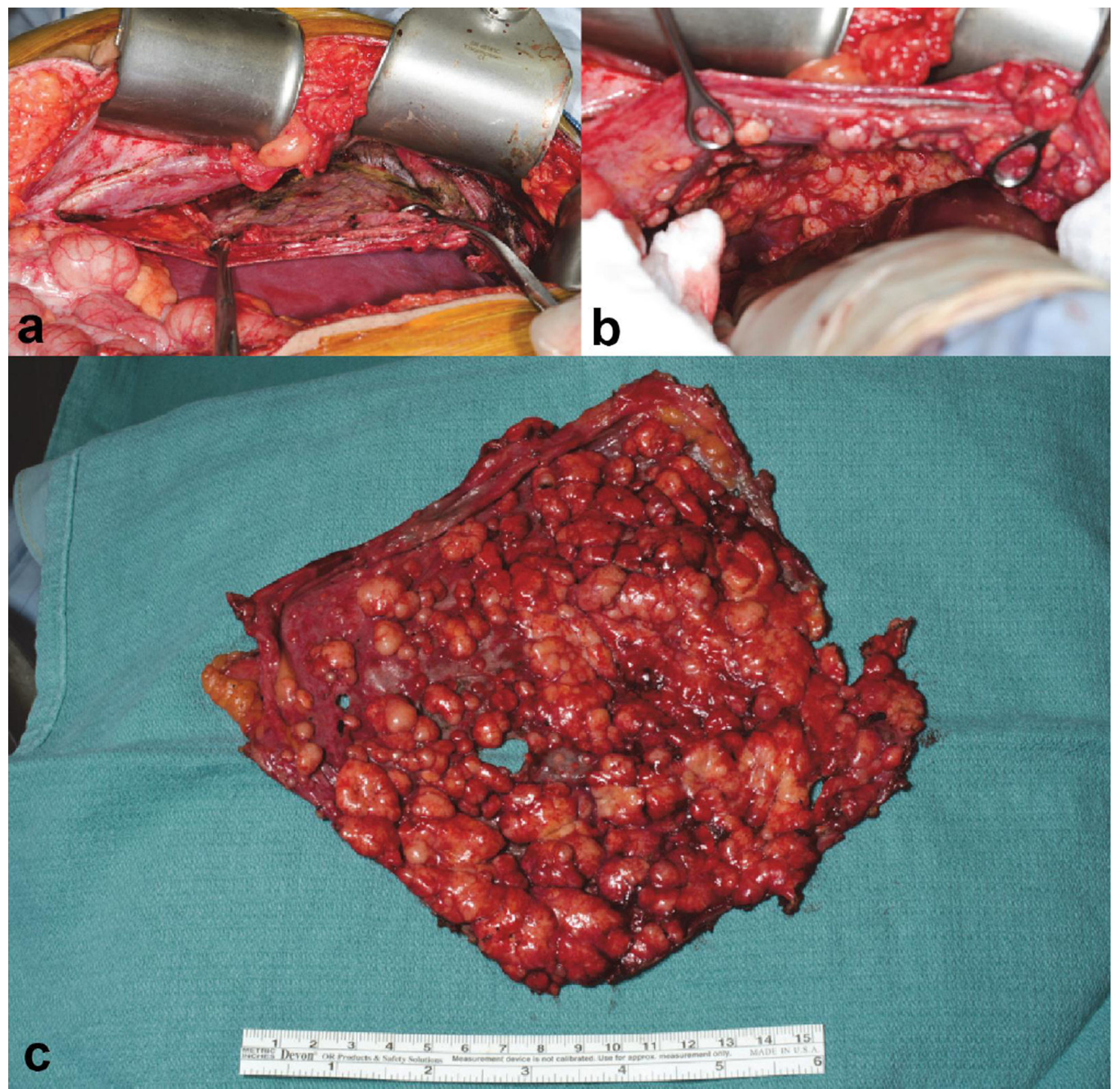

Figure 3.

A 'sheet' of sarcomatosis from DSRCT in the right diaphragm peritoneum. Figure $a$ and $b$ show the intra-operative dissection of the right diaphragm peritoneum. The final result (c) is one 'sheet' of tumor without any diaphragm muscle removed. 


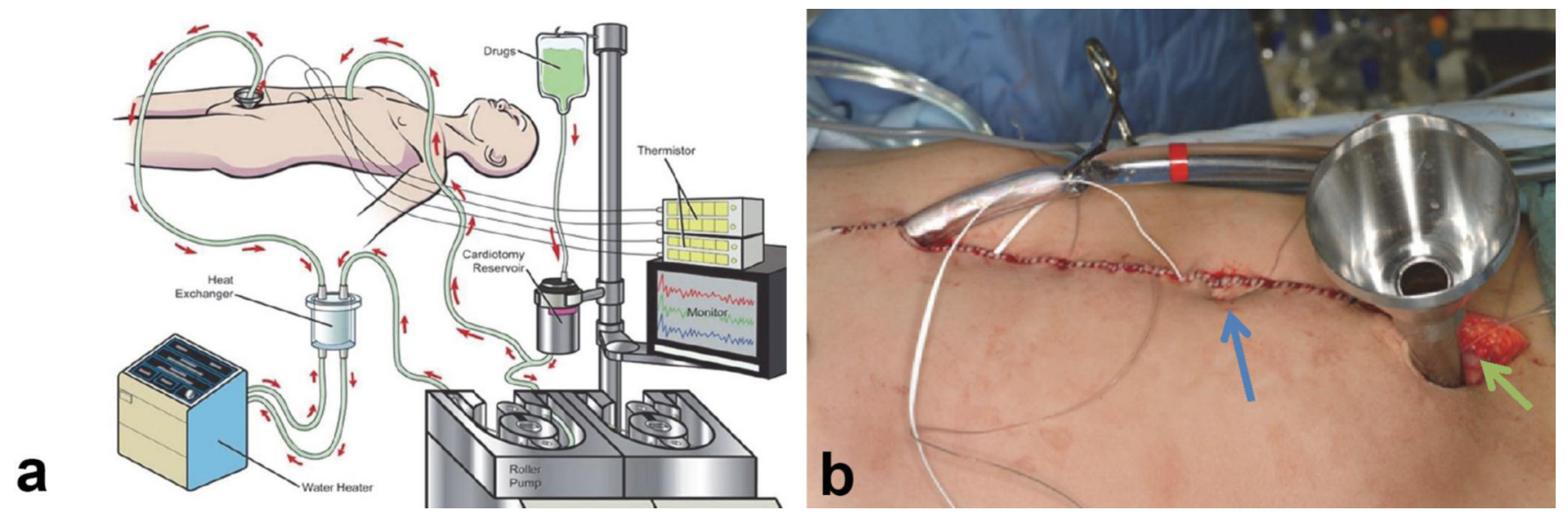

Figure 4.

(a) A representation of the HIPEC technique with a simple pump that pumps the heated chemotherapy $\left(41.5^{\circ} \mathrm{C}\right)$ into the abdominal cavity and recirculates, in a closed technique, over 90 minutes in the operating room, using cisplatin for chemotherapy in the case of DSRCT. (b) The closed abdomen of a patient after cytoreduction, ready to begin HIPEC. Temperature probes can be seen exiting from the midline skin closure, that will be attached to a computer to provide a constant monitoring of the intra-abdominal temperature. Black arrow denotes inflow cathete, green arrow is outflow port, and blue arrow is umilicus in a supine patient. 

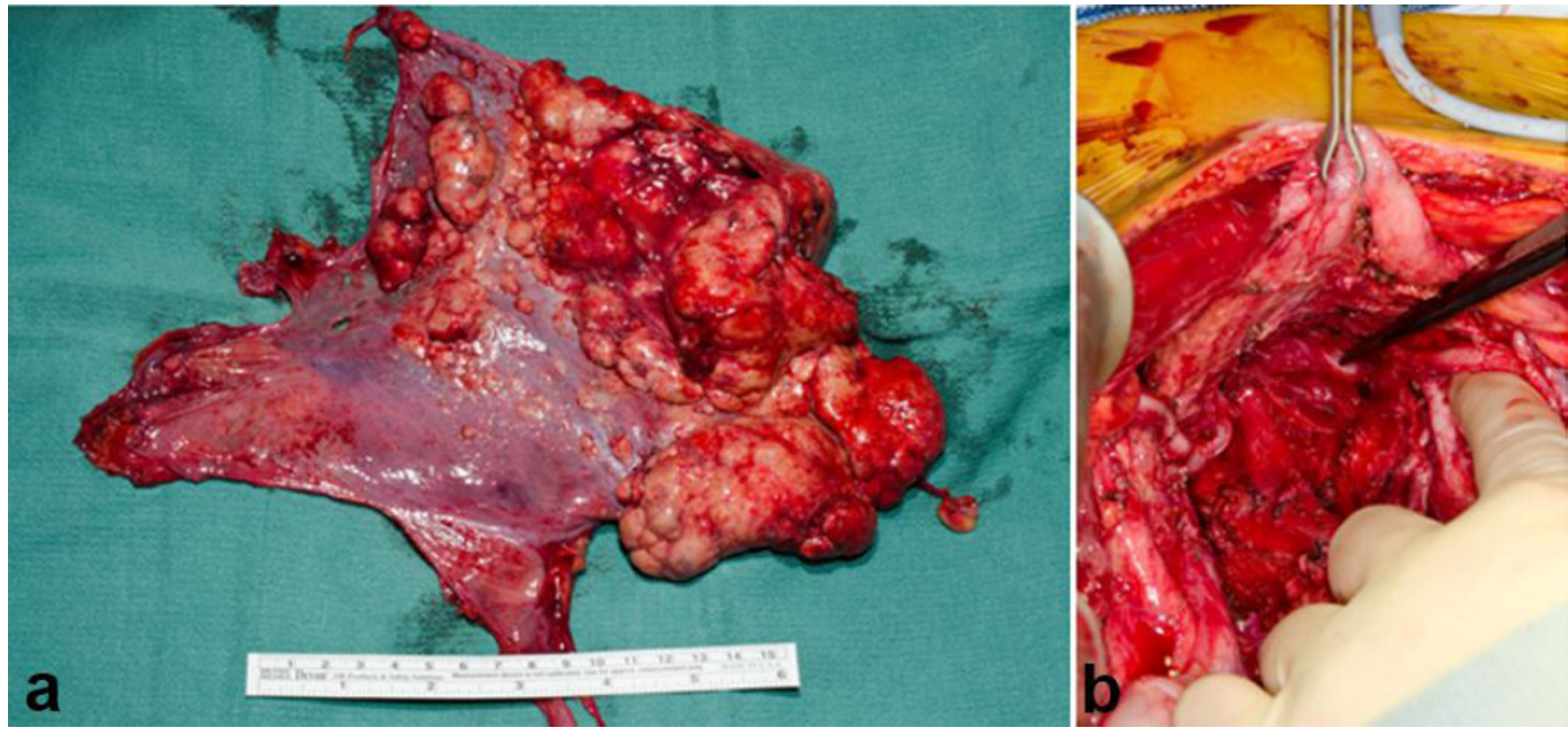

Figure 5.

(a) Pelvic peritonectomy in an 11-year-old male. (b) Appearance of pelvis after peritonectomy, demonstrating that the bladder, ureters, and vas deferens were spared, down to the seminal vesicles. 